\title{
Competencias Del Alumnado Formado En La Escuela De Formación De Profesorado: Una Investigación Reflexiva En El Contexto De Formación Inicial Docente
}

\author{
Maria Do Céu Pereira Antunes Da Silva \\ José Antonio Marín Marín \\ Esther Garzón Artacho \\ Universidad de Granada, España
}

doi: 10.19044/esj.2017.v13n7p68 URL:http://dx.doi.org/10.19044/esj.2017.v13n7p68

\begin{abstract}
This work was supported by the teaching practice. In it, we try to reflect on the output profile of the graduate student Teacher Training College. The article presented here includes a summary of the relevant aspects collected from a descriptive study, by applying a questionnaire to the student trainees. We conducted interviews with the governing body and some teachers. In order to talk about the profile it was necessary to analyse carefully the beliefs, opinions and performance of teaching, based on the following issue: will the students trained in Teacher Training College have an ideal output profile capable of exercise with pedagogical mastery the teaching profession? We have formulated the following general and specific objectives. General: To reflect on the output profile and the needs of the training offer that graduate students from T.T.C receive in terms of quality, skills, adaptation, and the different requirements on the models required by the Ministry of Education.
\end{abstract}

Keywords: Output profile; Teaching practice; Quality training; Skill; teaching Initial formation

\section{Resumen}

Este trabajo parte de la base del contexto de la formación del alumnado en prácticas formados en de la Escuela de Formación de Profesorado de Angola. Procuramos describir el perfil de salida de los educandos formados en la institución, sabiendo que son los futuros docentes del país. Se trata de un estudio descriptivo en el que se ha aplicado un instrumento de corte cuantitativo, como es el cuestionario al alumnado en 
prácticas. También se realizaron una serie de entrevistas a los cuerpo directivos y algunos profesores de la institución escolar. Para conocer y realizar una descripción del perfil del alumnado ha sido necesario analizar profundamente las creencias, opiniones y el desempeño de la actividad docente. Por tanto, partimos del problema en el que se plantea si los alumnos formados en la Escuela de Formación de Profesores poseen un perfil ideal para ejercer con maestría sus funciones como profesor. Una vez establecido el objeto de estudio se plantea como objetivo general el describir y reflexionar sobre el perfil de salida del alumnado, así como conocer las necesidades en la oferta de formación de la Escuela y su adaptabilidad a los criterios establecidos por el Ministerio de Educación.

Palabras clave: Calidad de formación, competencias, formación inicial docente, etapa pedagógica, educación superior

\section{Introducción}

El perfil profesionalizador que adopta el individuo en su práctica laboral es de suma importancia y se considera como el conjunto de capacidades técnicas, profesionales e interdependientes. Estos elementos se encuentran relacionados intrínsecamente con ciertas áreas de que intervienen en el aprendizaje, como son la cognitiva, la afectiva y la psicomotora.

De acuerdo con el Programa de Prácticas Pedagógicas implantado en la Escuela de Formación de Profesores (2005), para el desarrollo de una base cimentada y la formación de una profesionalidad sólida, los estudiantes se someten a la fase de practicas docentes a lo largo de su formación de manera gradual y teniendo la oportunidad de una formación inicial, esto conlleva a una participación plena y progresiva en situaciones de enseñanza-aprendizaje reales y aplicadas.

Según Pasos y Veiga (2004, p.15) “cuando se trata la práctica docente como formación inicial funciona como una herramienta para la ejecución del proceso de enseñanza-aprendizaje de manera eficiente y de calidad". En este contexto, las practicas docentes en la escuela de formación de profesores permiten desarrollar las habilidades profesionales en los estudiantes e inician el camino correcto para la adquisición de competencias docentes que tendrán que materializar en su futuro como profesores.

Estamos de acuerdo con la idea de Fialho, Silva y Zaragoza que defienden que "aparte de un proceso social del individuo, la formación es a la vez un proceso de aprendizaje centrado en la facilitación de la integración socio-laboral en el mercado”. (2013, p.11). En palabras de Antunes (2001, p.19), "la educación es un proceso de crecimiento que, con el objetivo de la auto-realización personal, profesional y social, se da a lo largo de toda la 
vida de los seres humanos y debe primar en todos los contextos en los que se desenvuelven".

En nuestro ámbito de actuación específico, la Escuela de Magisterio, las prácticas pedagógicas se inician en el grado 11. En este primer momento, el estudiante se limita a establecer un contacto primario con la institución escolar, donde observan y analizan la labor desempeñado por el tutor de aula; también conocen la institución sobre aspectos concernientes a la organización y gestión escolar. En un segundo momento, equiparado a la impartición del grado 12, comienzan a impartir clase en momentos cortos de tiempo y empiezan a participar en las reuniones de coordinación y otros eventos relacionados con la escuela y siempre acompañados por el tutor de prácticas de la institución escolar.

Este proceso finaliza en el grado 13, cuando se integra por completo en la vida escolar. En este caso, asume plenamente las funciones inherentes a la función docente.

En palabras de Pérez (2009) la enseñanza tiene una parte individual cuando requiere que cada maestro debe movilizar un abanico amplio de saberes, habilidades, técnicas, metodologías y competencias; sin embargo, su demostración dependerá del tiempo, del contexto, de los educandos y del propio docente.

En nuestra opinión, siguiendo la palabras de Marqués (2003), la educación es una de las acciones y actividades que comprenden mas nobleza, ya que se da la existencia de una relación entre el aprendizaje de los individuos y el bienestar social. La formación de los futuros profesionales educativos, junto con los gobiernos centrales, debe mantener una interrelación de cooperación con el objetivo de la mejora de la calidad educativa, desarrollando una formación integral y sostenible basada en la democracia del país.

La calidad educative se erige como un tema crucial en las sociedades contemporáneas. En un mundo globalizado, marcado por la innovación tecnológica que evoluciona de manera constante, no existe cabida para escuelas ineficientes y que proporcionen una enseñanza de mala calidad a los maestros o los encaucen hacia la desmotivación.

Por lo tanto, es de suma importancia conocer y establecer la duración de la formación inicial del estudiante para que se de su integración en la escuela de manera paulatina; dado que en nuestro país hay escuelas de formación de profesorado que no poseen una institución escolar adjunta para que se realicen estas prácticas docentes.

Un maestro es un agente que influye de manera directa en el entorno en el que vive y la sociedad en general, mediante su papel participativo y active. Por tanto, no es menos importante o representativa la contribución que puede llegar a hacer el alumno en prácticas, ya que no hay un perfil 
establecido de buen profesional educativo, pero sí hay buenas prácticas que mantienen un equilibrio entre la tradicionalidad y la innovación.

\section{La formación del profesorado en el $1^{\circ}$ Ciclo de Enseñanza Secundaria en Angola}

El tema central de esta investigación, como ya hemos comentado, es precisamente la formación de los maestros. El desarrollo de las habilidades, destrezas, capacidades y conocimientos tienen que darse a través de cursos de formación y desde las propias instituciones de formación de profesores. En el caso de las instituciones de educación superior se debe llevar a cabo una implantación de modelos y métodos que ayuden a dotar al alumnado de la suficiente autonomía y de una actitud crítica y reflexiva sobre su propia labor docente y los programas educativos que se llevan a cabo en el contexto angolano.

El sistema educativo debe tender al desarrollo armónico e integral del individuo, desde el nacimiento del individuo y hasta el final de su existencia.

En Angola, la Escuela de Formación de Profesorado es una institución dedicada, única y exclusivamente, a la formación de los maestros que desempeñarán la labor docente en las escuelas del ciclo de enseñanza secundaria, divididas en tres cursos $\left(7^{\circ}, 8^{\circ}\right.$ y $9^{\circ}$ grado) y atendiendo alumnos entre los 15 y 17 años de edad.

Actualmente, la Escuela de Formación del Profesorado incluye la formación específica ligada a cada especialidad que responde a los planes de studio vigentes en el primer ciclo de la secundaria. Sin embargo, se ha detectado que un numeroso grupo de profesores mantienen carencias formativas en dicha formación específica y otros docentes no tienen una formación pedagógica suficiente. A esto se une que el reclutamiento de los docentes, en ocasiones, se realiza sin que estos tenga una base sólida en su formación inicial en ciertas áreas y no llevan a cabo cursos de especialización antes de iniciar su práctica como profesor.

En cuanto a la gestión del propio proceso de formación del profesorado aparecen una multitud de dificultades que ocasiona que el perfil de los futuros maestros, una vez salen de la facultad, no sea aún el idóneo; todo ello si se reflexiona sobre los objetivos y metas establecidas desde las escuelas normales.

La institución de educación superior presenta ciertas problemáticas que hay que analizar y a las que es imprescindible dar respuesta de manera inmediata. Por un lado, en la Escuela de Formación del Profesorado existe una ratio alumno-profesor demesiado elevada. Espacios de gran importancia como: bibliotecas, laboratorios, instalaciones deportivas, comedor escolar, centro medico, etc., son inexistentes o se encuentran en condiciones precarias. Por otro lado, los programas educativos no están estandarizados y 
estructurados y se asevera una gran falta de recursos educativo. No existe una inspección escolar que se de regularmente y la vigilancia de los supervisores educativos han desembocado en una calidad educativa ínfima, donde el proceso de enseñanza-aprendizaje no es significativo y ha perjudicado al rendimiento académico de los estudiantes.

Haciendo hincapié en la situación actual de los planes de estudios implantados, decir que ofrecen una instrucción repetitiva, reproductiva y compartimentada, lo que dificulta que el alumnado que recibe la enseñanza no se ve como el eje central del aprendizaje, anulando el compromiso como futuro profesor.

Según Oliveira (2014), aunque no es posible relacionar un proyecto de formación de profesorado específico con características exclusivas de la profesión, puede afirmarse categóricamente que una formación de calidad debe proponerse formar a partir de un dominio riguroso del conocimiento, practicar el área de conocimiento específica en la que trabajará como maestro y la integración de los aspectos didácticos-pedagógicos. Los atributos de un profesional deben basarse en y desde una actitud crítica como ciudadano, active y participante de la vida cotidiana de su entorno y, también, como profesional educativo debe ser consciente que está proporcionando un servicio social destinado al bien común.

Para Formosinhos (2009), la formación del profesor se produce tanto de manera directa e indirecta a lo largo del curso escolar. El aprendiz durante la formación se enfrenta a la propia práctica pedagógica, partiendo de sus experiencias y conocimientos previos, reaprendiendo y actualizándose a lo largo de los años formativos y profesionales.

\section{Criterios de selección del profesorado angolano para el ingreso en las instituciones escolares}

La contratación de personal educativo es promovida por los concursos públicos convocados por la Dirección Provincial de Educación, en base a los conocimientos adquiridos, las denominadas oposiciones. El propio Ministerio de Educación promueve la adquisición de competencias y es el encargado de preparar al personal para la labor educativa a desarrollar, específicamente a través de los gobiernos locales. Todo ello dirigido al profesorado en plantilla de los centros escolares.

Para el alumnado que se forma en la Escuela de Profesorado, una vez elegida la especialidad y habiéndose inscrito, acceden a través de una prueba escrita. Se seleccionan aquellos con mayor expediente académico y priorizando a los más jóvenes.

Darling-Hammond (citado por Lopez y Silva, 2010), concluye que es posible preparar al futuro profesorado antes de que se integren definitivamente en el sistema educativo del país, es decir, mientras se están 
formando. Para ello, el autor identifica pormenorizadamente una serie de referencias, entre ellas destacamos: la consistencia de una visión común y clara sobre la enseñanza, la interrelación entre conocimientos, habilidades, creencias y valores; un código ético y deontológico de la profesión y, por ultimo, un plan de estudios basado en el desarrollo integral del individuo.

El enseñar bien requiere conocimiento y experiencia pedagógica, pero también, y no menos importante, tener inteligencia emocional. Siguiendo a Estanqueiro (2012), los docentes competentes buscan promover y potenciar las diferentes habilidades del alumnado a través de metodologías diversas, recursos diferentes y la utilización de instrumentos de evaluación que muestren evidencias de aprendizaje que demuestren la evolución del proceso de aprender.

\section{Vocación y exigencias para la profesión docente en Angola}

Las escuelas normales de Angola son aquellas que sirven como alternativa a muchas personas que tienen dificultades para entrar en las instituciones de preferencia primaria, ya que existen factores que intentan eliminar sesgos como el azar y no aceptar a estudiantes que no tienen vocación docente real.

Dentro de esta realidad, también se encuentran profesionales que, ante la imposibilidad de encontrar empleo dentro de su profesión especifica, deciden comenzar los estudios de magisterio.

Hoy en día, las escuelas están iniciando un reajuste de programaciones y planes de estudio para elevar la calidad de la enseñanza angolana y atender al contexto real social, cultural, económico y político del país. Por ende, se dan dos corrientes claras entre los docentes formados previamente y los actuales; se ocasiona una escisión sobre la propia visión sobre la enseñanza. A esto se une que aquellos docentes que tienen una dilatada experiencia profesional no fueron formados en las instituciones de formación de profesores, sino que realización cursos de capacitación y adaptación pedagógica en los centros de la zona.

De acuerdo a la vision de Zau (2012), es indispensable diseñar un plan general de formación de profesorado en el que se definan las características y el perfil que los profesores deberían tener, así como de otros agentes educativos, partiendo de la base de que cualquier acto educativo es una intervención social y en el que es preciso dotar a las escuelas de unos recursos humanos competentes.

\section{Método}

\section{Población, muestra e instrumentos}

El estudio presentando en este artículo forma parte de una investigación de mayor envergadura. Se ha partido de una metodología de 
investigación de naturaleza descriptiva y no experimental, ya que tratamos de conocer y describir un suceso educativo en un momento determinado e inscrito en un contexto específico; en este caso, analizar el perfil docente del alumnado en prácticas formado en la Escuela de Formación del Profesorado de Benguela, Angola.

El instrumento utilizado ha sido de corte cuantitativo, un cuestionario diseñado y construido ad hoc, validado por juicio de expertos, y que quedó conformado por seis dimensiones que engloban ítems sobre el perfil personal y académico (A), la relevancia y utilidad de los elementos del proceso de enseñanza-aprendizaje en la formación inicial (B); la relevancia del acompañamiento del profesor-tutor (C); la relevancia de la intervención del profesor-mentor (D); la satisfacción del grado de organización y gestión de las actividades del alumnado en prácticas (E); el saber pedagógico adquirido en la Escuela de Formación de Profesorado de Benguela (F) y la la percepción de las competencias adquiridas y el perfil de salida del alumnado (G).

Para el análisis y tratamiento de los datos obtenidos del cuestionario, fue utilizado el paquete estadísticos para ciencias sociales (SPSS) v.22 para Windows. El coeficiente de alfa de Conbrach obtenido fue $>.90$, valor excelente que confirma la validez del cuestionario.

Los participantes fueron seleccionados por un muestreo aleatorio simple de una población total de 2.615 alumnos, quedando representado por 350 estudiantes interinos. En cuanto al género, encontramos que el 39,4\% eran hombres y el 60,6\% mujeres. Atendiendo a la especialidad los estudiantes representan el 20\% en Matemáticas y Física, Lengua Portuguesa y Educación Moral y Cívica, Historia y Geografía, Biología y Química y, por ultimo, el 20\% son alumnos en prácticas del Magisterio Primario.

\section{Resultados}

Partiendo de los objetivos propuestos en el estudio, presentamos a continuación los resultados más relevantes de la investigación.

Con la intención de conocer la opinión del estudiantado sobre la relación con la práctica de la formación llevada a cabo en la Escuela de Formación de Profesores de Benguela y también desde las escuela de acogida del alumnado en prácticas, concluimos que el 48,9\% permanece de acuerdo respecto a la conexión entre la institución de educación superior y la práctica docente cotidiana. Por otro lado, el 43,1\% afirmó estar en completo acuerdo. Las prácticas equiparán a los docentes de las herramientas necesarias para su desarrollo profesional. 


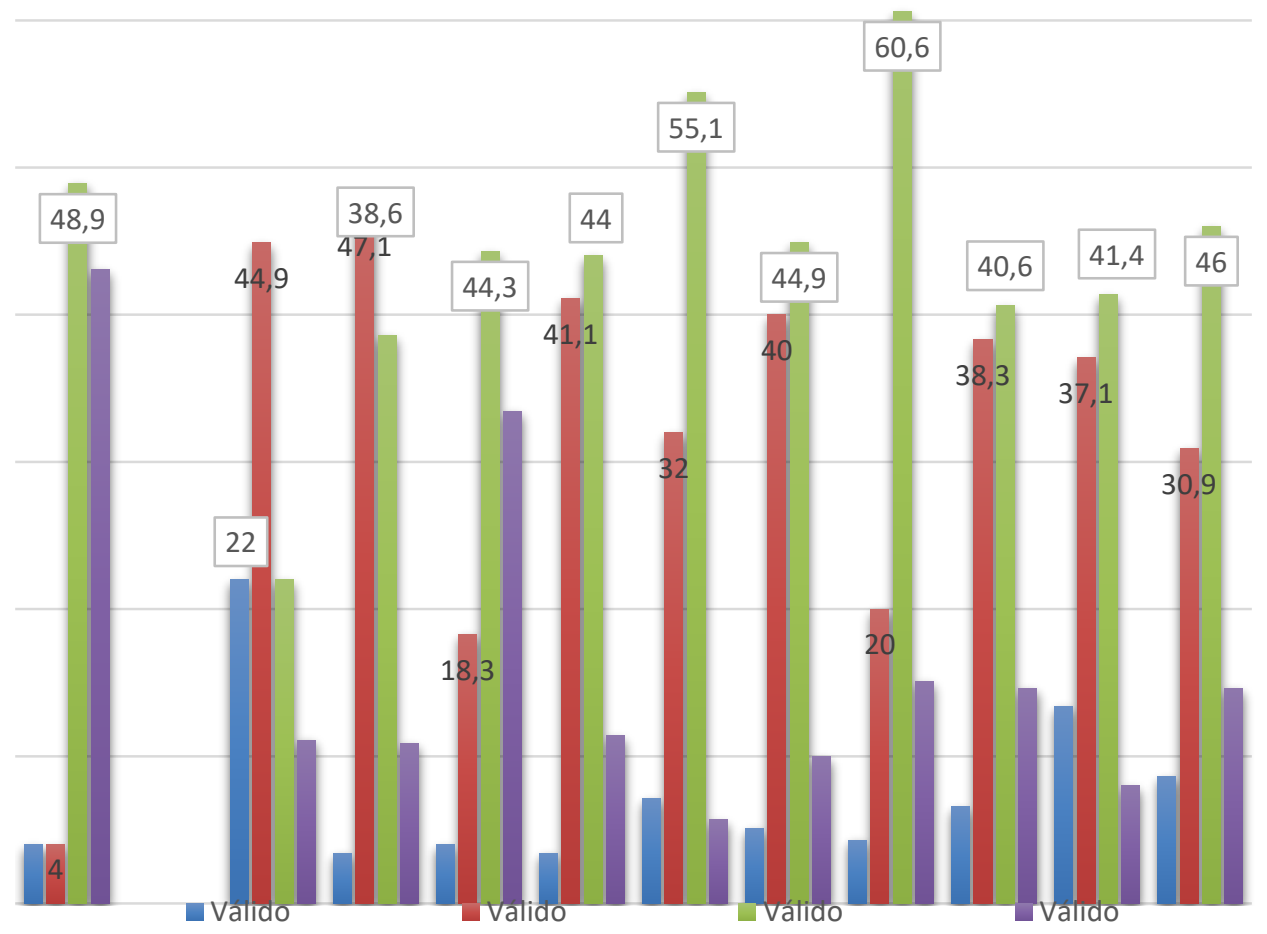

Figura 1. Gráfico porcentual relevancia y utilidad formativa del periodo de prácticas

En la Figura 2 se atiende a la percepción y opinión del alumnado sobre si el maestro-tutor del centro escolar demuestra disponibilidad y atención, el 45,1\% del alumnado participante afirma su desacuerdo, frente al $35,7 \%$ que permanece de acuerdo. Estos resultados nos llevan a percibir cierta banalización de la profesión docente, la falta de motivación e interés tanto del profesorado acompañante como de los receptores de la formación. En cuanto a si la Escuela de Formación del Profesorado atendía adecuadamente a los estudiantes, el 45,7\% permanece de acuerdo y el 40,9\% en pleno acuerdo. 
A figura do professor tutor é positiva para...

Cada vez mais, a escola de aplicação é...

O professor tutor tem demonstrado...

Os argumentos utilizados pelo professor...

A integração e o intercâmbio de.

O professor tutor tem facilitado a.

O professor tutor exerce influência positiva..

A direcção da escola de aplicação tem.

O professor tutor dá possibilidades aos.

O grau de autonomia e o desenvolvimento...

A relação com o professor tutor tem...

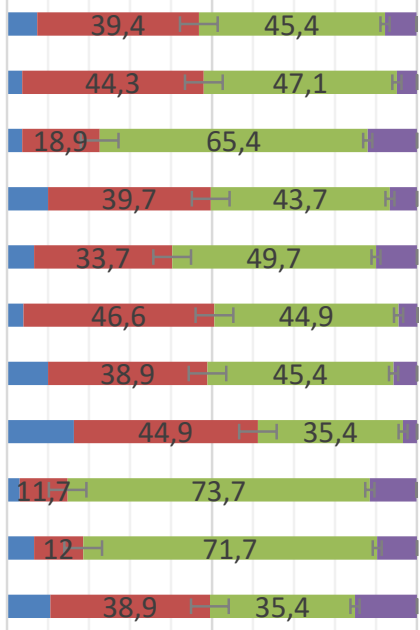

0

50

Figura 2. Gráfico porcentual relevancia del acompañamiento del profesor tutor

En la Tabla 1 podemos observas los resultados obtenidos tras realizar la prueba estadística chi-cuadrado de Pearson que establece las diferencias estadísticamente significativas entre la variable de agrupación género y algunos de los ítems del cuestionario aplicado. Asumimos que existirá relación de dependencia o asociación entre las variables cuando $\mathrm{p} \leq, 05$ con un grado de confianza de $95 \%$.

Tabla 1. Resultados prueba de chi-cuadrado de Pearson con variable agrupación género

\begin{tabular}{ccc}
\hline & \multicolumn{2}{c}{$\begin{array}{c}\text { Variable agrupación } \\
\text { Género }\end{array}$} \\
\cline { 2 - 3 } & $\mathrm{N}$ & $\mathrm{gl}$ \\
\hline $\begin{array}{c}\mathrm{X}^{2} \\
\text { As práticas pedagógicas contribuem para a formação profissional } \\
\text { dos futuros professores. }\end{array}$ & 350 & 3 \\
\hline $\begin{array}{c}\text { Com a prática pedagógica os formandos aprendem } \\
\text { procedimentos para manter a disciplina na aula }\end{array}$ & 350 & 3 \\
\hline $\begin{array}{c}\text { A direcção da escola de aplicação tem orientado e coordenando as } \\
\text { práticas pedagógicas }\end{array}$ & 350 & 3 \\
\hline
\end{tabular}




\begin{tabular}{|c|c|c|c|}
\hline $\begin{array}{l}\text { O professor de prática pedagógica segue o trabalho dos } \\
\text { formandos durante o desenvolvimento das aulas práticas, } \\
\text { demonstrando disponibilidade e atenção }\end{array}$ & 350 & 3 & ,025 \\
\hline Os alunos adquirem os saberes ao nível da prática pedagógica & 350 & 3 & ,006 \\
\hline $\begin{array}{l}\text { As políticas das escolas de formação de professores são } \\
\text { implementadas segundo as exigências do mercado }\end{array}$ & 350 & 3 & ,000 \\
\hline
\end{tabular}

En cuanto a la opinión del alumnado participante en referencia a si las prácticas contribuyen a su formación como futuros docentes, se muestra que el $30,3 \%$ de las mujeres se encuentran en complete acuerdo con la afirmación, frente al 25,7\% de los hombres. En el grado de acuerdo destaca el $23,1 \%$ masculino, mientras que son ellas las que obtienen menor representación con el 12,9\%. Se concluye que el alumnado ve importante esta formación práctica durante el transcurso de sus estudios para la adquisición de competencias docentes.

En la Figura 4 se muestra que los alumnos consideran que las prácticas permiten desarrollar procedimientos para el mantenimiento de la disciplina en el aula. En el grado de acuerdo destacan los estudiantes de género masculino con el 23,7\%, mientras que ellas afirman estar de acuerdo en un 20,6\%. En el grado de total acuerdo, son las estudiantes con un 22,6\% las más representadas, al igual que en el grado de desacuerdo con un 14,3\%.

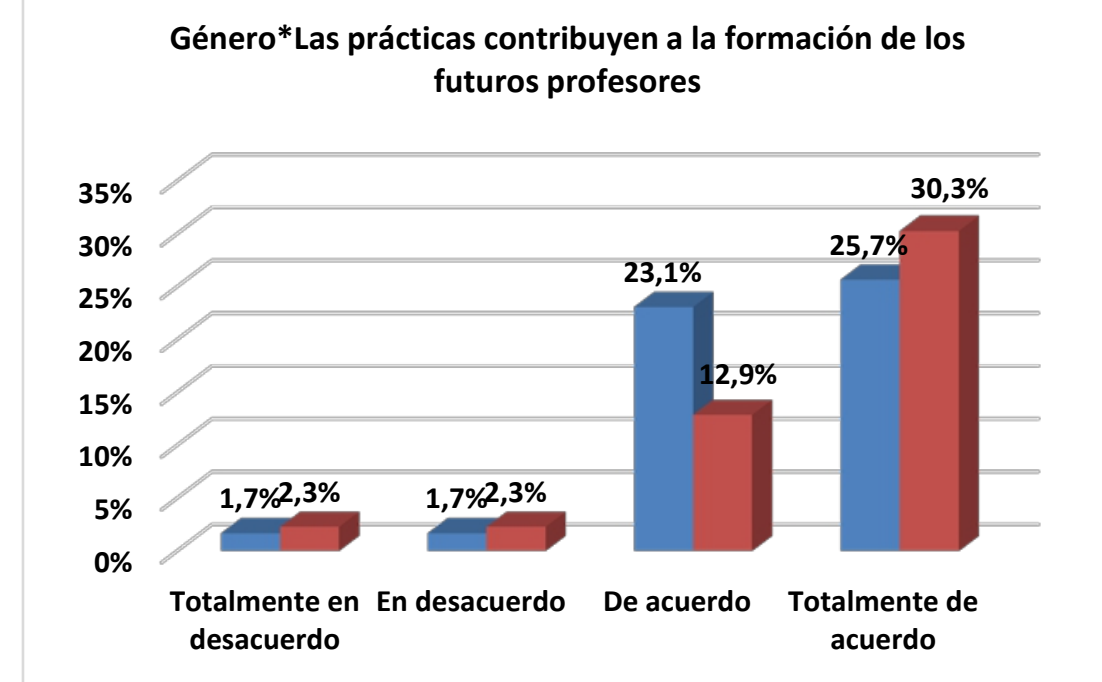

Figura 3. Gráfico comparativo género*las prácticas pedagógicas contribuyen a la formación de los futuros profesores. 


\section{Género*Con las prácticas docentes el alumnado aprende procedimientos para mantener las disciplina en el aula}

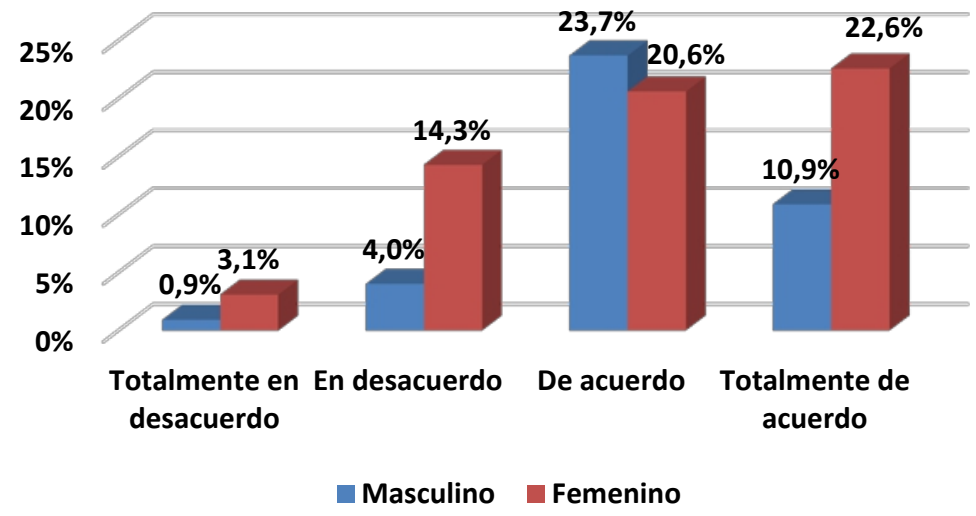

Figura 4. Gráfico comparative Género*Las prácticas docentes permiten desarrollar procedimientos para mantener la disciplina en el aula.

En cuanto a la coordinación y la orientación de los centros durante el periodo de prácticas se muestra que el $11,4 \%$ de las mujeres se encuentran en complete desacuerdo y el 23,4\% en desacuerdo, siendo ellas las que perciben una menor calidad en cuanto a estos aspectos llevados a cabo por los centro escolares.

\section{Los centros escolares de acogida de alumnado mantiene una coordinación y orientación en las prácticas}

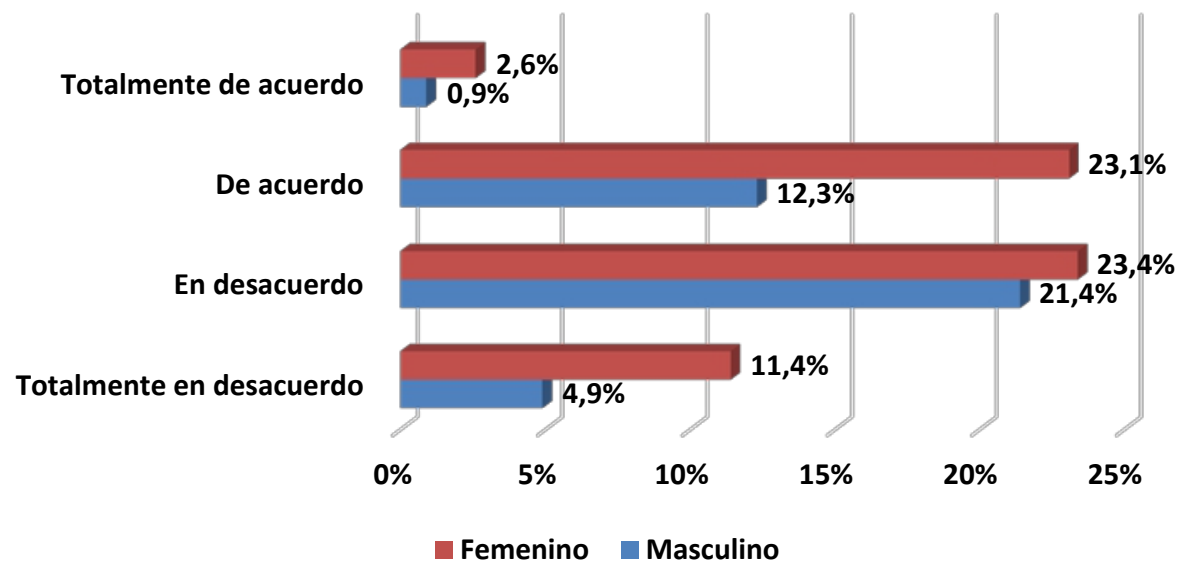

Figura 5. Género*Los centros escolares de acogida de alumnado mantiene una coordinación y orientación en las prácticas

En referencia a la tabla 2 se observa que sobre si el alumnado adquiere conocimiento gracias al periodo de prácticas se observa que los 
estudiantes de género femenino tienen un mayor acuerdo con el 42,3\%, frente al 28\% de alumnos de género masculino.

Así mismo, respecto a si las políticas que se implantan en las escuelas de formación del profesorado se interrelacionan con las exigencias del mercado laboral y la sociedad, son ellos los que mantienen un mayor desacuerdo con la afirmación, en el grado de total de acuerdo el género masculino representa un 24,3\%; en el grado de desacuerdo destacan las mujeres con el 25,1\%, mientras que ellos se mantienen con un 6,6\%.

Tabla 2. Porcentajes Cruces variable agrupación género

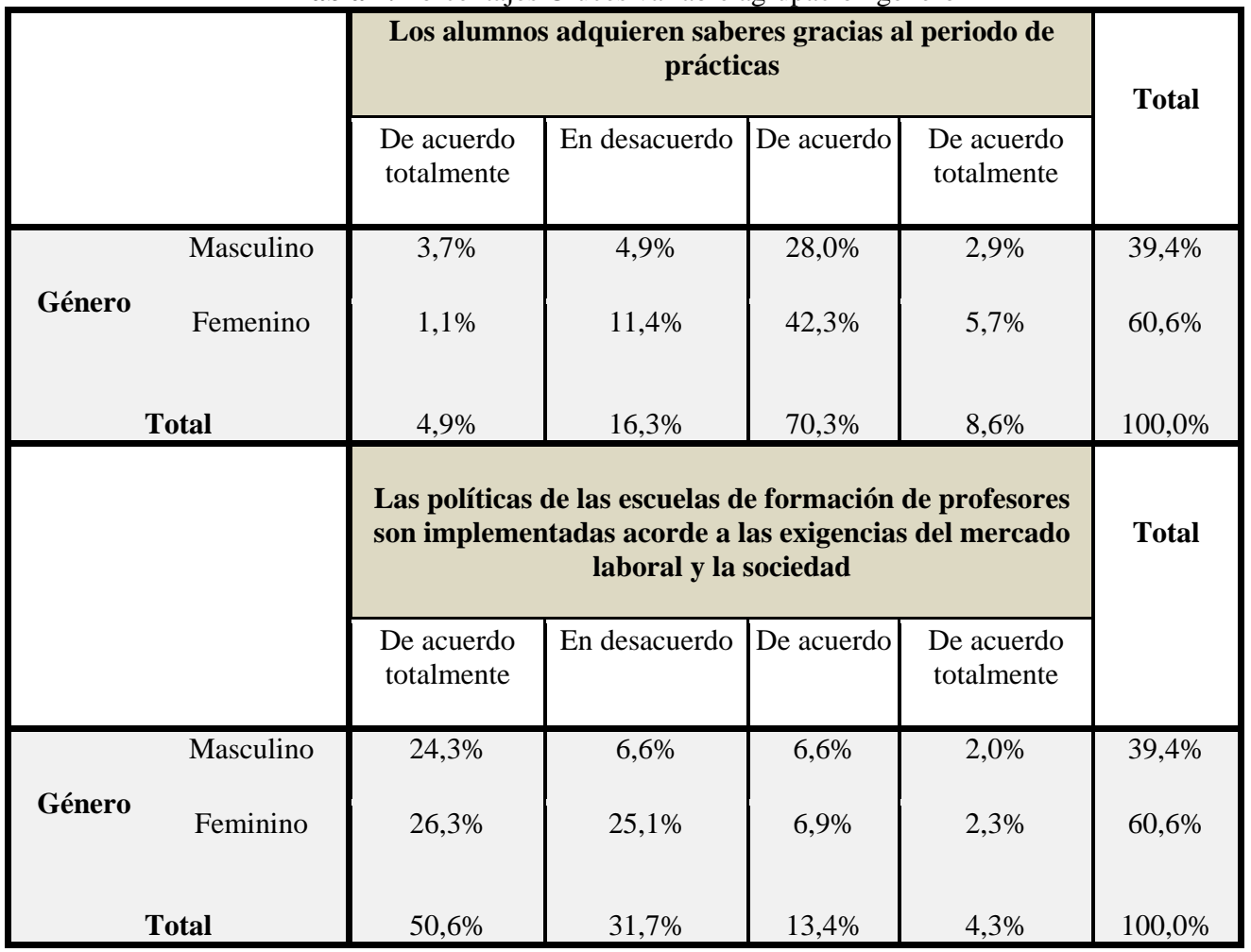

\section{Conclusion}

Los resultados analizados y expuestos anteriormente no han llevado hacia una serie de conclusions. Primero, la falta de escuelas que acojan a alumnado en prácticas se convierte en una de las problemáticas más urgentes de solucionar, ya que pone en peligro la formación inicial de los futuros docentes.

Segundo, la falta de formación de los profesores-tutores de las escuela puede provocar situaciones de disminución de rendimiento académico por parte de los estudiantes y una carencia en la adquisición de competencias docentes para su futuro profesional. Las escuelas de magisterio deben movilizar todos los recursos necesarios, ya sean materiales, humanos y 
de infraestructuras, y centrarse en el desarrollo de las habilidades de los estudiantes, logrando que movilicen una serie de saberes, habilidades, valores y actiudes para poder resolver las problemáticas que puedan llegar a surgir en su práctica diaria.

Tercero, la escuela debe investigar y estudiar el contexto real de la comunidad para establecer las demandas y necesidades que surgen de ella, para así poder adaptar e implantear políticas educativas que calen en los estudiantes y en su formación. Unimos a esto, que las instituciones de formación dle profesorado de repensar y reestructurar los criterios de acceso y admisión de los estudiantes, partiendo del sendero del bien común de la sociedad y la educación como derecho y deber por y para todos.

Por ultimo, deben establecerse relaciones de cooperación y colaboración más estrechas entre el profesorado-tutor de la escuela y el profesor de acompañamiento de la institución superior, la conexión es indispensable para dotar esta formación inicial de globalidad e interconexión entre la teoría y la práctica docente diaria.

\section{References:}

1. Antunes, M. C. P. (2001). Teoria e prática pedagógica. Lisboa. Piaget editora.

2. Estanqueiro, A. (2012). Boas práticas na educação. O papel dos professores. Lisboa. Editorial Presença.

3. Fialho, J.; Silva, C. A. \& Saragoça, J. (2013). Formação profissional. Práticas organizacionais, políticas públicas e estratégias de acção. Lisboa. Edições Sílabo.

4. Formosinho J. (coord) (2009). Formação de professores. Aprendizagem profissional e acção docente. Portugal. Porto editora.

5. Lopes, J. \& Silva, H. S. (2010). O professor faz a diferença. Porto. Edições técnicas.

6. Marques, R. (2003). Motivar os professores. Lisboa. $1^{\text {a }}$ Edição. Editorial presença.

7. Oliveira, M. R. N. S. (2014). Professor: formação, saberes e problemas. Portugal. Porto editora.

8. Passos \& Veiga (2004). A prática pedagógica do professor de didáctica. Campina São Paulo. $8^{a}$ Edição: papirus editora.

9. Pérez, J. F. B. (2009). Coaching para docentes. Motivar para o sucesso. Portugal. Porto editora.

10. Programas de Metodologia, Práticas, Seminários e Estágios Pedagógicos, da Escola de Formação de professores. (2005). Luanda

11. Zau, F. (2012). Do acto educativo ao exercício da cidadania. Angola. $1^{\mathrm{a}}$ Edição. Edições Angola. 